\title{
Uncertainties in tidal theory: Implications for bloated hot Jupiters
}

\author{
Jérémy Leconte ${ }^{1}$, Gilles Chabrier ${ }^{1}$ and Isabelle Baraffe ${ }^{1,2}$ \\ ${ }^{1}$ École Normale Supérieure de Lyon, 46 allée d'Italie, F-69364 Lyon cedex 07, France; \\ Université Lyon 1, Villeurbanne, F-69622, France; CNRS, UMR 5574, Centre de Recherche \\ Astrophysique de Lyon; \\ (jeremy.leconte, chabrier, ibaraffe@ens-lyon.fr) \\ ${ }^{2}$ School of Physics, University of Exeter, Stocker Road, Exeter EX4 4PE, UK
}

\begin{abstract}
Thanks to the combination of transit photometry and radial velocity doppler measurements, we are now able to constrain theoretical models of the structure and evolution of objects in the whole mass range between icy giants and stars, including the giant planet/brown dwarf overlapping mass regime (Leconte et al. 2009). In the giant planet mass range, the significant fraction of planets showing a larger radius than predicted by the models suggests that a missing physical mechanism which is either injecting energy in the deep convective zone or reducing the net outward thermal flux is taking place in these objects. Several possibilities have been suggested for such a mechanism:

- downward transport of kinetic energy originating from strong winds generated at the planet's surface (Showman \& Guillot 2002),

- enhanced opacity sources in hot-Jupiter atmospheres (Burrows et al. 2007),

- ohmic dissipation in the ionized atmosphere (Batygin \& Stevenson 2010),

- (inefficient) layered or oscillatory convection in the planet's interior (Chabrier \& Baraffe 2007),

- Tidal heating due to circularization of the orbit, as originally suggested by Bodenheimer, Lin \& Mardling (2001).

Here we first review the differences between current models of tidal evolution and their uncertainties. We then revisit the viability of the tidal heating hypothesis using a tidal model which treats properly the highly eccentric and misaligned orbits commonly encountered in exoplanetary systems. We stress again that the low order expansions in eccentricity often used in constant phase lag tidal models (i.e. constant $Q$ ) necessarily yields incorrect results as soon as the (present or initial) eccentricity exceeds $\sim 0.2$, as can be rigorously demonstrated from Kepler's equations.
\end{abstract}

Keywords. planets and satellites: general

\section{Uncertainties in tidal theory}

Tidal heating has been suggested by several authors to explain the anomalously large radius of some giant close-in observed exoplanets (Bodenheimer et al. 2001; Jackson, Greenberg \& Barnes 2008; Miller, Fortney \& Jackson 2009; Ibgui, Spiegel \& Burrows 2011). The basic scenario consists in a planet left on a wide, very eccentric orbit by an early event during its formation. The orbit then slowly decays due to tidal dissipation, leading to a circularization on a timescale of a few Gyr's. This slow circularization, however, is due to the fact that all these authors use a tidal model based on a quasi circular approximation, truncated at $2^{\text {nd }}$ order in eccentricity.

This quasi circular approximation, developed initially to study the tidal evolution of the solar system planets (Goldreich \& Soter 1966; Ferraz-Mello, Rodríguez \& Hussmann 
2008), which have negligible eccentricities, is valid only in this very limit, $e \ll 1$. In the context of exoplanetary systems, current high eccentricities are common and initial high eccentricities are very likely, as inferred from non-transiting planets observed by radial velocity. Therefore, this quasi circular approximation is no longer correct, as demonstrated in Eggleton et al. (1998), Wisdom (2008) and Leconte et al. (2010) and summarized below.

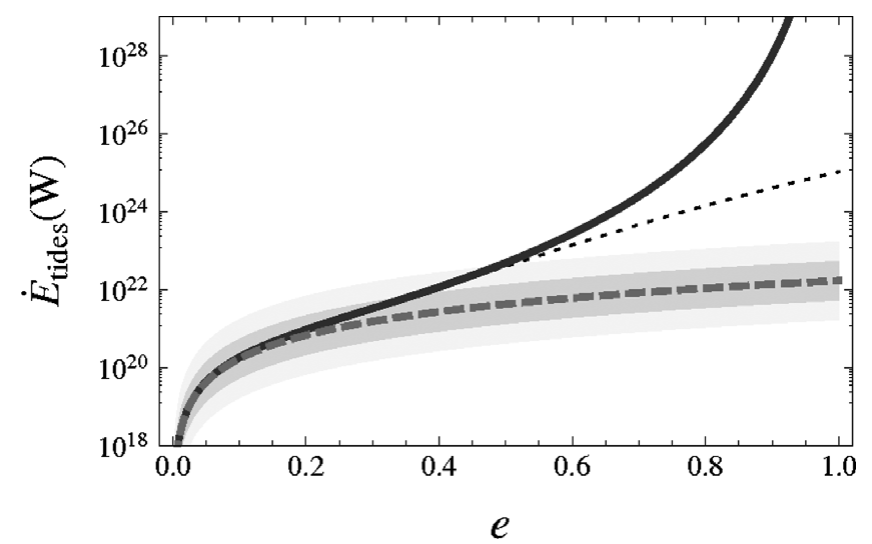

Figure 1. Tidal energy dissipation rate in a pseudo-synchronized planet as a function of the eccentricity calculated with the complete formula (curve), with the $e^{2}$-truncated formula (dash) and to $e^{10}$ (Dotted). The ratio of the two curves only depends on the eccentricity and not on the system's parameters. The inner (outer) shaded area shows the uncertainty in the heating when allowing the dissipation parameter to vary within one (two) order of magnitude. The actual values were derived using HD 209458 b parameters.

Present analytical theories for tidal interaction are all based on the equilibrium tide and weak friction approximation, since no adequate theory for dynamical tides presently exists. These theories differ in two ways

- (i) their parametrization of the dissipative processes. The most common prescriptions are either a constant phase lag (constant- $Q$ ) model or a constant viscosity or time lag (constant- $\Delta t$ ) model.

- (ii) their mathematical treatment of the geometry of the orbits: perturbative developments around the coplanar/circular keplerian orbits or closed formulae, valid for any eccentricity.

While these two sources of differences between tidal models are completely different by nature, they are often, erroneously, mixed together. Indeed, only the constant time lag model, because of the linear dependence of the phase lag upon the time lag in this model, allows the calculations to be carried out in terms of closed formulae for any eccentricity. High order calculations in eccentricity in the framework of the constant- $Q$ model are very cumbersome (see Ferraz-Mello et al. 2008).

As demonstrated by Wisdom (2008) and Leconte et al. (2010), even though large uncertainties remain on the quantification of the dissipative processes themselves, the discrepancies arising from the differences in the treatment of the orbital geometry at moderate to high eccentricities $(e \gtrsim 0.2-0.3)$ can become dominant by orders of magnitude. This is summarized on Fig. 1 which compares the tidal heating given by the constant time lag model of Leconte et al. (2010) (solid curve) and by the quasi circular approximation of Peale \& Cassen (1978) (dashed curve). In comparison, the inner (outer) shaded area illustrate the impact of the uncertainty in the heating when allowing the tidal dissipation 
parameter to vary by one (two) order of magnitude. For $e>0.4$, we see that high order terms in $e$ yield a contribution which is larger than the uncertainty in the quantification of the dissipative processes. Such a behavior at high eccentricity is well understood in the context of celestial mechanics and is due to the slow convergence of elliptical expansion series (Danjon 1980; Cottereau, Aleshkina \& Souchay 2010). Using the admitedly large uncertainty in tidal dissipative processes as an argument to use necessarily wrong orbital equations is thus by no means justifiable.

Therefore, calculations based on constant- $Q$ models truncated at the order $e^{2}$ cannot be applied to (initial or actual) eccentric orbits larger than about 0.2-0.3, a common situation among detected exoplanetary systems. This implies a major caveat in previous calculations coupling thermal and orbital evolutions. In particular, as illustrated in Fig. 1, using a $e^{2}$-truncated model leads to a severely underestimated tidal dissipation timescale at large eccentricity, and thus to an overestimated amount of dissipated tidal energy in exoplanet interiors at present ages (Leconte et al. 2010).

\section{Tidal heating and bloated exoplanets.}

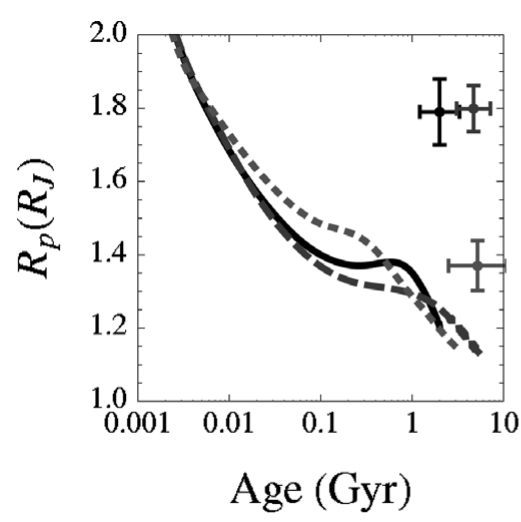

(a) Planetary radius

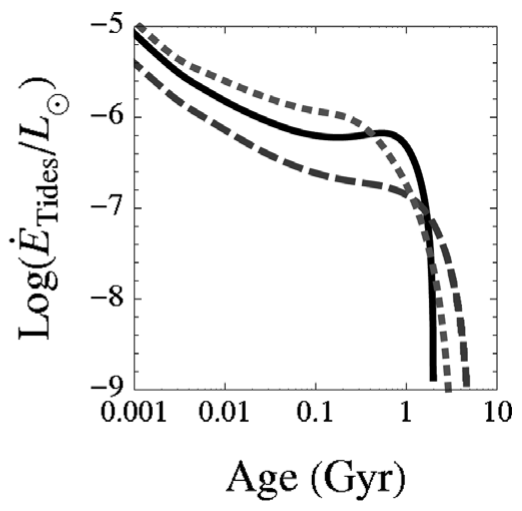

(b) Tidal energy dissipation

Figure 2. Evolutionary tracks for WASP-12 b (solid), TrES-4 b (dashed) and WASP-4 b (dotted) that lead to the best agreement with the observed orbital parameters for these systems. These runs assume $Q_{0, \mathrm{p}}^{\prime}=10^{6}$ and $Q_{0, \star}^{\prime}=10^{6}$. Tidal dissipation is not sufficient to sustain the large radii observed for these planets.

Revisiting the viability of the tidal heating hypothesis to explain the anomalously large Hot Jupiter radii $\dagger$ with the Hut complete tidal model, Leconte et al. (2010) showed that, although tidal friction indeed provides a non negligible contribution to the planet heat content and can possibly explain some transiting system large radius, the tidal heating hypothesis fails to explain the radii of extremely bloated planets like - among others - HD 209458 b, TrES-4 b, WASP-4 b or WASP-12 b, as illustrated on Fig 2. These conclusions have been confirmed recently by Hansen (2010). The main reason is the early circularization of the orbit of these systems, as mentioned above, which thus yields insufficient heating at late epochs. Note that these conclusions rely on the assumption of

$\dagger$ Numerical values of the radius anomaly of transiting planets as defined in Leconte et al. 2010 can be found at perso.ens-lyon.fr/jeremy.leconte/JLSite/JLsite/Exoplanets_Simulations.html. 
a genuine two body system. The presence of a third body able to excite eccentricity in a massive giant planet for several gigayears would provide an other explanation. Accurate observations are necessary to support or exclude the existence of such undetected close low-mass or distant massive companions.

\section{Acknowledgements}

This work was supported by the Constellation european network MRTN-CT-2006035890, the french ANR "Magnetic Protostars and Planets" (MAPP) project and the "Programme National de Planétologie" (PNP) of CNRS/INSU. We acknowledge the use of the www.exoplanet.eu database. J.L. wishes to thank L. Cottereau for insightful discussions concerning current developments in Celestial Mechanics.

\section{References}

Batygin, K. \& Stevenson, D. J. 2010, ApJ, 714, L238

Bodenheimer, P., Lin, D. N. C., \& Mardling, R. A. 2001, ApJ, 548, 466

Burrows, A., Hubeny, I., Budaj, J., \& Hubbard, W. B. 2007, ApJ, 661, 502

Chabrier, G. \& Baraffe, I. 2007, ApJ, 661, L81

Cottereau, L., Aleshkina, E., \& Souchay, J. 2010, A\& A, 523, A87

Danjon, A. 1980, Astronomie generale. Astronomie spherique et elements de mecanique celeste, ed. Danjon, A.

Eggleton, P. P., Kiseleva, L. G., \& Hut, P. 1998, ApJ, 499, 853

Ferraz-Mello, S., Rodríguez, A., \& Hussmann, H. 2008, Celestial Mechanics and Dynamical Astronomy, 101, 171

Goldreich, P. \& Soter, S. 1966, Icarus, 5, 375

Hansen, B. M. S. 2010, ApJ, 723, 285

Ibgui, L., Spiegel, D. S., \& Burrows, A. 2011, ApJ, 727, id.75

Jackson, B., Greenberg, R., \& Barnes, R. 2008, ApJ, 681, 1631

Leconte, J., Baraffe, I., Chabrier, G., Barman, T., \& Levrard, B. 2009, A\&A, 506, 385

Leconte, J., Chabrier, G., Baraffe, I., \& Levrard, B. 2010, AESA, 516, A64+

Miller, N., Fortney, J. J., \& Jackson, B. 2009, ApJ, 702, 1413

Peale, S. J. \& Cassen, P. 1978, Icarus, 36, 245

Showman, A. P. \& Guillot, T. 2002, A\&A, 385, 166

Wisdom, J. 2008, Icarus, 193, 637 